\title{
Monitoria proativa: uma experiência didático-pedagógica do grupo de ensino de Geologia Introdutória da Faculdade de Geologia da Universidade Federal do Pará, Belém-PA
}

\author{
Francisco de Assis Matos de Abreu \\ Faculdade de Geologia, Instituto de \\ Geociências/UFPA famatos@ufpa.br \\ José Fernando Pina Assis \\ Faculdade de Geologia, Instituto de \\ Geociências/UFPA \\ josepina@ufpa.br \\ Jamylle Trindade de Matos \\ Graduanda do Curso de Geologia/UFPA \\ jamylletrindade-01@hotmail.com \\ Leticia Briglia Ramos Arantes \\ Graduanda do Curso de Geologia/UFPA \\ leticiabriglia@yahoo.com.br \\ Eliã Jéssica Oliveira Silva \\ Graduanda do Curso de Geologia/UFPA \\ eliajessica.s@hotmail.com
}

\begin{abstract}
PROACTIVE MONITORING: A DIDACTIC AND PEDAGOGICAL EXPERIENCE OF THE INTRODUCTORY Geology TeaChing Group of the Geology FaCulty of PARÁ Federal University, Belém, PA. This paper presents the first results of the Proactive Monitoring Project, implemented in 2009 at the College of Geology, Federal University of Pará, as a pedagogical tool for student use in optimizing the content of the introductory geologies, translated into two instructional products: Geology Fieldwork Didactic Guide and Didactic-Methodologic Kits on Introductory Geology. Furthermore, the paper describes in general terms, the educational process of their development. Citation: Abreu F.A.M.de, Assis J.F.P., Matos J.T.de, Arantes L.B.R., Silva E.J.0. 2014. Monitoria Proativa: uma experiência didático-pedagógica do Grupo de Ensino de Geologia Introdutória da Faculdade de Geologia da Universidade Federal do Pará (Belém, Pará-Brasil) Terræ Didatica, 10(3):378-382. http:// www.ige.unicamp.br/terraedidatica/.
\end{abstract}

KEYWORDS: proactive monitoring, introductory geology, fieldwork.

RESUMO Este trabalho apresenta os primeiros resultados concretos do Projeto Monitoria Proativa, implementado em 2009 no Curso de Geologia da Universidade Federal do Pará, como ferramenta pedagógica para otimização do aproveitamento estudantil nos conteúdos das geologias introdutórias, traduzidos em dois produtos didáticos: Roteiro de Atividades para Prática de Campo em Geologia Introdutória e Kits Didático-Metodológicos em Geologia Introdutória. Além disso o trabalho descreve, em linhas gerais, o processo pedagógico de sua elaboração. 


\section{Introdução}

A participação de estudantes graduandos no processo da efetiva construção do conhecimento científico tem aumentado muito nos últimos tempos. Hoje em dia, a prática tornou-se comum em todos os setores da academia e seu principal objetivo é o despertar da postura investigativa mediante a incorporação definitiva do processo epistêmico (Severino, 2013).

Em consonância, o Grupo de Ensino de Geologia Introdutória do Curso de Geologia da UFPA desenvolve desde 2009, um projeto de inserção estudantil na prática da produção integral do conhecimento em geologia, que envolve quatro conteúdos do Projeto Pedagógico do Curso.

Juntos, os conteúdos se apresentam desmembrados em disciplinas de atividades teórico-práticas e em seu afazer, apresentam uma nova estratégia de abordagem metodológica para potencializar os primeiros contatos dos graduandos com as geociências, notadamente aqueles relativos aos trabalhos de campo. Contemplam as disciplinas Introdução às Geociências (com sua complementar Prática de Campo em Introdução às Geociências) e Geologia Geral (com sua complementar Prática de Campo em Geologia Geral).

Este trabalho apresenta os primeiros resultados concretos do projeto, traduzidos em dois produtos didáticos: Roteiro de Atividades Didáticas para Prática de Campo em Geologia Introdutória e Kits Didático-Metodológicos em Geologia Introdutória, e descreve em linhas gerais o processo de construção e estruturação dos mesmos.

\section{A monitoria}

O envolvimento de estudantes de graduação em procedimentos sistemáticos da produção do conhecimento é o caminho mais adequado para o alcance dos objetivos da própria aprendizagem. Para (Severino, 2013) aprender é necessariamente uma forma de praticar o conhecimento apropriando-se de seus processos constituintes específicos de tal sorte, que o fundamental seja, não seu produto, mas o processo.

Implantada em 2009, a experiência da Monitoria Proativa tem sido fator determinante no aproveitamento estudantil, expresso na qualidade do rendimento estudantil nas avaliações finais das disciplinas. Para sua implantação e operacionalização foi decisivo o suporte da Plataforma Moodle, instrumento digital institucional utilizado pela

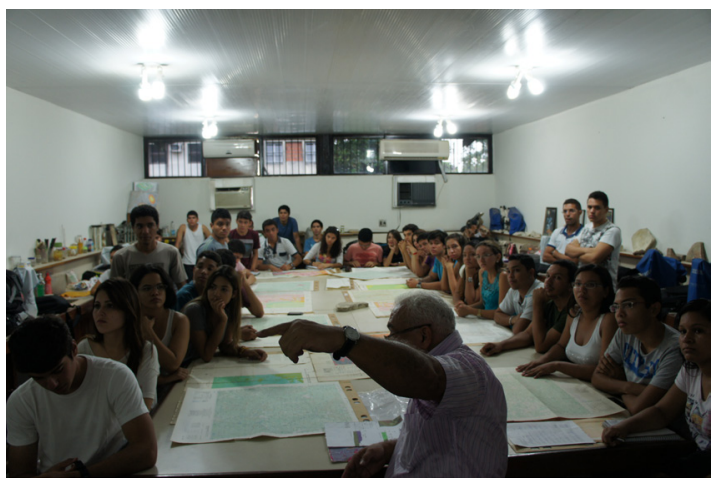

Figura 1. Atividade de cartografia geológica, preparatória para saída a campo com os estudantes da disciplina

Assessoria de Educação a Distancia (AEDI/UFPA) e vinculado aos programas de educação da Universidade Aberta do Brasil (UAB), no qual desde 2005 está inserido o Programa de Pós-graduação lato sensu do Instituto de Geociências.

A estrutura organizacional contempla a agregação de 12 (doze) estudantes do $3^{\circ}$ período letivo da matriz curricular do Curso de Geologia, selecionados com base na aferição do desempenho acadêmico em disciplinas anteriormente cursadas, e subjetivamente a partir da demonstração da capacidade de liderança, facilidade de inter-relacionamento com colegas, além da clara manifestação de interesse em participar da experiência pedagógica.

As atividades preparatórias (Figuras 1 e 2) incluem discussões coletivas sistemáticas entre professores tutores e estudantes monitores, para ajustes no funcionamento integrado das disciplinas. Incluem: atualização permanente dos conteúdos-programa; leituras dirigidas de itens temáticos específicos; elaboração preliminar dos roteiros de atividade para aulas práticas; apoio tutorial a estudantes nas atividades de sala de aula e de campo; avaliação orientada de exercícios teóricos e práticos.

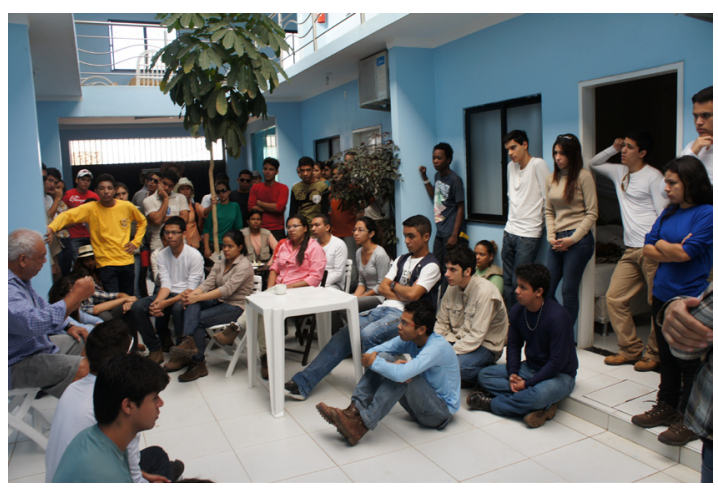

Figura 2. Preleção da coordenação do projeto para dois grupos de monitores, como parte do treinamento para as atividades de campo 


\section{A tutoria}

Para (Eliasquevici e Fonseca, 2004) a ação tutorial é "mediação que pode ser desempenhada em contextos diversos e nas diferentes formas". Segundo as autoras, tanto presencialmente como à distância, é fundamental a prática dos "plantões tutoriais" programados ou eventuais, onde atuam professores tutores e estudantes monitores.

No caso concreto, desde sua implantação as atividades de tutoria são realizadas em grupo, com um tutor para cada equipe de 5/6 estudantes trabalhando em três espaços de abordagem: 1- Na página eletrônica das disciplinas, abrigada na Plataforma Moodle, que disponibiliza textos temáticos e vídeos documentais em mídia digital, além de exercícios avaliatórios das disciplinas; 2- No ambiente de sala de aula (Figura 3) com orientação durante as atividades práticas; 3- Nas atividades de campo (Figura 4) com acompanhamento permanente em todos os momentos de trabalho.

Ainda citando Eliasquevici e Fonseca (op cit) a mediação é processual e considerando cada situação, tutores e monitores se apropriam de multimeios disponíveis, para transformar objetivos em práticas pedagógicas concretas.

Assim, cada grupo de monitores é responsável pelo rendimento das equipes de estudantes sob sua orientação e trabalha em regime de interdependência em relação aos demais grupos. Após as atividades letivas, o grupo de monitores reúne com os tutores para discussão e reorientação didática visando a preparação para a atividade do dia seguinte.

\section{Os produtos}

Um dos objetivos concretos do programa de monitoria proativa é traduzido pela produção de material didático-metodológico (kits didáticos e roteiros para realização de atividades práticas de campo), inteiramente elaborados pelo grupo de monitores, sob orientação dos tutores.

KITS DIDÁTICOS - A produção desse material de suporte didático composto por minerais, rochas e fósseis, traduz-se em atividade de mão-dupla, pedagogicamente estimulante tanto para os monitores (Figura 5), como para estudantes das disciplinas envolvidas.

A produção inicia ainda no campo, com a seleção e coleta feitos especificamente para os objetivos consequentes do projeto. No laboratório, as amostras passam por triagem para escolha dos litotipos

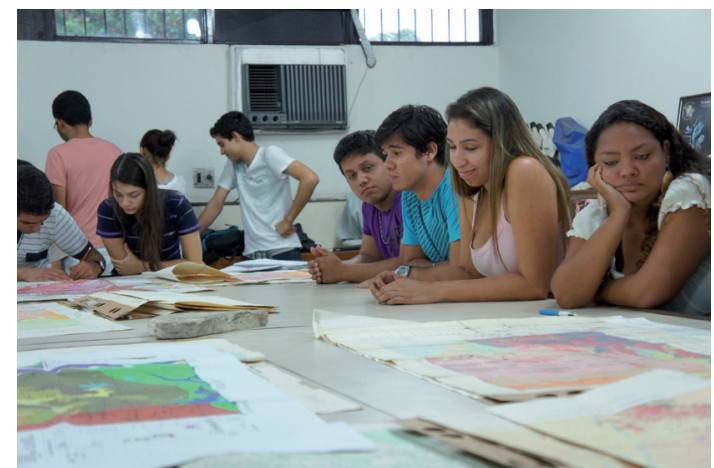

Figura 3. Treinamento didático-pedagógico do grupo de monitores em atividade sobre cartas geológicas;

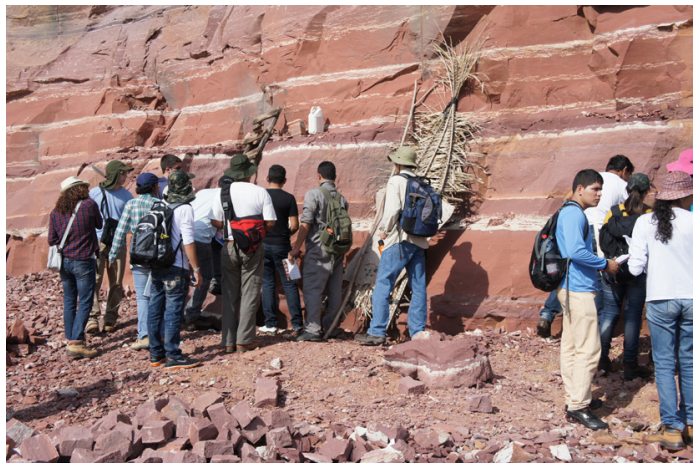

Figura 4. Atividades práticas orientadas, realizadas pelos monitores em situação concreta de campo com estudantes da disciplina Geologia Geral.

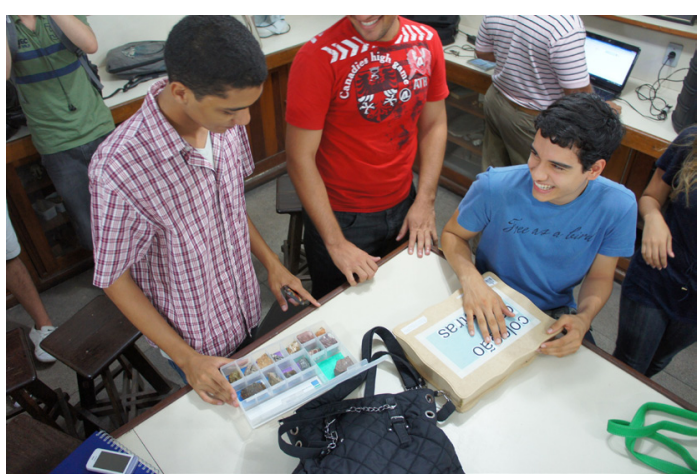

Figura 5. Atividade prática de demonstração com utilização dos kits didáticos;

diagnósticos. Ao tempo em que são instrumentados pelos tutores (Figura 6) os monitores auxiliam os estudantes das turmas na elaboração dos kits, cujos mostruários são artesanalmente confeccionados com utilização de material reciclado (plástico, madeira e papelão).

ROTEIRO PARA O TRABALHO DE CAMPO - O Roteiro de atividades para Prática de Campo em Geologia Geral foi concebido para potencializar o aproveitamento dos estudantes nas disciplinas que contemplam atividades de campo no 


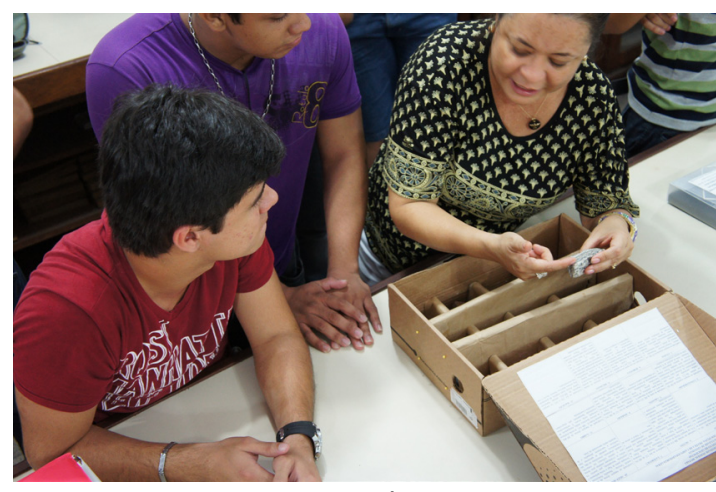

Figura 6 - Treinamento didático dos monitores, sob orientação de uma tutora de Petrologia;

Curso de Geologia. Sua elaboração contou com o resgate do material produzido pelos próprios estudantes do curso nas viagens de campo anteriores (relatórios de viagem, mapas, seções geológicas e anotações em cadernetas de campo) e segue três objetivos:

1. qualificação técnica de graduandos de Geologia e pedagógica no caso dos graduandos das licenciaturas em Ciências Naturais, Geografia e Biologia, para exercício no magistério de conteúdos geológicos nos níveis de ensino fundamental e médio;

2. suporte metodológico para apoio às atividades pedagógicas do programa de pós-graduação lato sensu da Faculdade de Geologia da UFPA, que contemple alunos oriundos de diversas áreas do conhecimento, grande parte sem conhecimentos básicos de geologia;

3. preparação da base conceitual para implantação do Curso de Licenciatura Plena em Geociências no âmbito do Instituto de Geociências da UFPA.

O texto trata de forma objetiva e sucinta de temas relativos à prática de campo não apenas das disciplinas Introdução às Geociências e Geologia Geral. Seu ponto central inclui informações conceituais das unidades geológico/geomorfoló- gicas de âmbito regional, notadamente aquelas que traduzem grandes eventos formatadores da Plataforma Sul-americana de (Almeida, 1967) e (Almeida et al 1977).

No caso específico, o roteiro sumariza informações sobre as Províncias Estruturais da Borborema, do Parnaíba e Costeira e contempla descrição e roteirização de seções e perfis geológicos, instrumentos fundamentais tanto para a realização dos trabalhos de campo como para elaboração do relatório final da viagem.

\section{Resultados}

Cinco anos após seu início, o projeto apresenta valores importantes, traduzidos não apenas na evolução dos números de graduandos atendidos, mas e especialmente, nos números de monitores preparados, se considerado os 40 ingressos no exame vestibular anual. Isto permite concluir que nesse breve tempo vigência o projeto preparou 52 monitores, bem mais que uma turma inteira de ingressantes anuais no curso de geologia (Quadro 1).

\section{Conclusões}

O Projeto Monitoria Proativa conta com apoio institucional e suporte instrumental do Laboratório de Cartografia Geológica - GEOCART, da Faculdade de Geologia da UFPA. Ainda que preliminares, os resultados são animadores e mostram claramente que foi acertado o caminho tomado pelo Grupo de Ensino de Geologia Introdutória. O grupo de 12 tutores/2012 atuou com os calouros/2013 e visivelmente ampliou sua qualificação, aferida pelos tutores em dois pontos pedagógicos considerados fundamentais:

1. Maior capacidade para interagir conceitualmente com os calouros, nas discussões sobre temas geológicos como raciocínio espaço-

Quadro 1 - demonstrativo absoluto/percentual dos resultados apresentados pelo projeto Monitoria Proativa no intervalo 2009-2013.

\begin{tabular}{|c|c|c|c|c|c|c|}
\hline INTEGRANTES/ANO & $\mathbf{2 0 0 9}$ & $\mathbf{2 0 1 0}$ & $\mathbf{2 0 1 1}$ & $\mathbf{2 0 1 2}$ & $\mathbf{2 0 1 3}$ & TOTAL \\
\hline TUTORES & 02 & $02(4 \%)$ & $03(5 \%)$ & $04(7 \%)$ & $03(5 \%)$ & $\mathbf{1 4}(\mathbf{4 , 8} \%)$ \\
\hline MONITORES & 08 & $\begin{array}{c}08 \\
(16 \%)\end{array}$ & $\begin{array}{c}12 \\
(18 \%)\end{array}$ & $\begin{array}{c}12 \\
(19 \%)\end{array}$ & $12(21 \%)$ & $\mathbf{5 2}(\mathbf{1 8} \%)$ \\
\hline ESTUDANTES & 40 & $\begin{array}{c}40 \\
(80 \%)\end{array}$ & $\begin{array}{c}52 \\
(78 \%)\end{array}$ & $\begin{array}{c}48 \\
(75 \%)\end{array}$ & $44(75 \%)$ & $\mathbf{2 2 4}(\mathbf{7 7 , 2} \%)$ \\
\hline TOTAL/ANO & $\mathbf{5 0}$ & $\mathbf{5 0}$ & $\mathbf{6 7}$ & $\mathbf{6 4}$ & $\mathbf{5 9}$ & $\mathbf{2 9 0}$ \\
\hline
\end{tabular}


-temporal (litoestratigráfico), petrogênese (ciclo das rochas) e evolução geológica das províncias estruturais visitadas durante os trabalhos de campo;

2. Maior discernimento nas discussões intragrupos, entre-grupos e com os tutores na fase de atividades de pós-campo e no acompanhamento mais técnico durante a preparação dos kits didáticos e do relatório final dos calouros;

Os próximos passos serão: 1 - Ampliação da base de apoio pedagógico com a implantação dos grupos de monitoria proativa 2014 (16 monitores); 2 - Reelaboração do Roteiro de Atividades para Prática de Campo; 3 - Criação do banco de dados geológicos de campo para suporte às atividades práticas do Curso de Geologia; 4 - Redefinição ao espectro pedagógico do grupo docente, com a criação Grupo de Docência Aplicada ao Ensino de Geociências.

\section{Referencial bibliográfico}

Almeida F.F.M.de. 1967. Origem e Evolução da Plataforma Brasileira. Rio de Janeiro, DNPM/DGM. 36p. (Bol. 241).

Almeida F.F.M.de, Hasui Y., Brito Neves B.B.de., Fuck R.A. 1977. Províncias estruturais brasileiras. In: Simp. Geol. Nordeste, 8, Campina Grande, 1977. Anais... Campina Grande, Soc. Bras. Geol., p. 363-391.

Eliasquevici M.K., Fonseca N.A. 2004. Educação à distância: orientações para início de um percurso. Ed. UFPA (EDUFPA). 93p.

Severino A.J. 2013. Metodologia do Trabalho Científico. 23 ed. Cortez Ed. 304p. 